\title{
ARBITRAGE, RATIONALITY, AND EQUILIBRIUM
}

\begin{abstract}
No-arbitrage is the fundamental principle of economic rationality which unifies normative decision theory, game theory, and market theory. In economic environments where money is available as a medium of measurement and exchange, no-arbitrage is synonymous with subjective expected utility maximization in personal decisions, competitive equilibria in capital markets and exchange economies, and correlated equilibria in noncooperative games. The arbitrage principle directly characterizes rationality at the market level; the appearance of deliberate optimization by individual agents is a consequence of their adaptation to the market. Concepts of equilibrium behavior in games and markets can thus be reconciled with the ideas that individual rationality is bounded, that agents use evolutionarily-shaped decision rules rather than numerical optimization algorithms, and that personal probabilities and utilities are inseparable and to some extent indeterminate. Risk-neutral probability distributions, interpretable as products of probabilities and marginal utilities, play a central role as observable quantities in economic systems.
\end{abstract}

Keywords: Bayesian decision theory, game theory, arbitrage, coherence, incompleteness, competitive equilibrium, correlated equilibrium, capital asset pricing model, risk-neutral probabilities.

\section{INTRODUCTION}

Bayesian decision theory, game theory, and competitive market theory rest on common foundational assumptions about the rationality of individual economic agents. These assumptions traditionally have been expressed as axioms on preference orderings (e.g., completeness, transitivity, etc.), implying that each agent possesses a well-defined subjective probability distribution and utility function and that her goal is the maximization of subjective expected utility. In situations such as games and markets, where two or more agents interact, additional assumptions about inter-agent consistency are imposed: e.g., the agents hold common beliefs about exogeneous events and their rationality is common knowledge. The collective behavior of agents is assumed to lead to a state of equilibrium in which each agent's position is optimal (expected-utility-maximizing) for her given the positions of 
the other agents, and the existence of such equilibria is proved by recourse to fixed-point theorems.

This idealized view of economic rationality is by no means universally accepted. The assumption that beliefs and preferences are completely ordered has been rejected by many decision theorists and philosophers (e.g., Koopman 1940, Good 1962, Smith 1961, Aumann 1962, Suppes 1974, Levi 1980); the decision-making "paradoxes" first pointed out by Allais $(1953,1971)$ and Ellsberg (1961) are still a lively topic of debate; and the proposition that rationality is bounded by information-processing limitations (Simon 1955, 1976) or by evolutionary considerations (Nelson and Winter 1982) has given rise to a "behavioralist" countermovement in economics. (For a dialogue between rationalist and behavioralist views, see Hogarth and Reder 1986.) An enormous body of psychological research has accumulated in the last few decades to establish that real agents can not - or will not - behave consistently as expected-utility maximizers even in laboratory experiments, inspiring a proliferation of theories of "nonexpected utility."

In this paper it will be argued that decision theory, game theory, and market theory can be placed on a more secure and parsimonious footing by appeal to the principle of no arbitrage rather than the usual ordering axioms on preferences. The arbitrage principle already has a long history: in the literature of Bayesian statistics and decision theory, it was introduced by de Finetti (1937) as a basis for defining subjective probability, resurfaced in debates between frequentist and Bayesian statisticians in the 1960's and 1970's, and has emerged again in recent arguments over the foundations of subjective expected utility (e.g, Yaari 1985, Seidenfeld 1988). In the finance literature, the arbitrage principle explicitly underlies the capital structure theorem (Modigliani and Miller 1958), the valuation of cash flows (Beja 1967, Rubinstein 1976, Ross 1978), and the pricing of assets and options (Black and Scholes 1973, Merton 1973, Ross 1976, Breeden and Litzenberger 1978, Brennan 1979). No-arbitrage is central to classical welfare economics, where it goes by the name of "Pareto optimality." In models of exchange economies, the relation between arbitrage and competitive equilibrium has been noted by Kreps (1981) and Werner (1987). More recently, the arbitrage principle has been proposed as a 
foundation for noncooperative game theory through its dual relation with the concept of correlated equilibrium (Nau and McCardle 1990, Nau 1991a), and also as a foundation for modeling trade under conditions of asymmetric information (Nau 1990). Yet, the avoidance of arbitrage is usually perceived as a mere adjunct to more basic assumptions about individual and collective rationality: a supporting argument for (rather than the fundamental axiom of) Bayesian decision theory, a by-product (rather than a definition) of equilibrium behavior in markets.

It will be shown here that no-arbitrage is the core principle of rationality which remains after economic models are stripped of their excess baggage of intrinsically unverifiable or empirically disconfirmed assumptions about the perfectness of human cognition, particularly the assumption that belief and preference orderings are complete. The arbitrage principle still provides support for the most important concepts of equilibrium in games and markets, but with weaker information requirements. It is consistent with "procedural" or "cognitive" views of rationality (e.g., Simon 1976; Munier 1991) which emphasize that the information-processing capabilities of the individual are finite and tailored to the specific demands of her physical and cultural environment.

The competitive market can be thought of as a decentralized computational device in which myopic individual behavior serves to allocate production and consumption through a process closely resembling the simplex algorithm of linear programming (Scarf 1990). We will show that in a variety of economic contexts the primal optimization problem "solved" by the market can be framed as the mere exploitation of arbitrage opportunities, and the corresponding dual problem establishes the existence of equilibrium probabilities, marginal utilities, and/or prices. This does not presume sophisticated optimizing behavior on the part of individual agents: more plausibly, they inherit and propagate informal decision-making rules by a process akin to natural selection. Rationality rubs off on the agent -i.e., she acquires the appearance of sophistication - through her adaptation to the market. Thus, Bayesians are made, not born.

The idea that predation by arbitrageurs will exert selective pressure in favor of "rational" agents, and that optimizing behavior should 
therefore be most apparent in highly arbitraged markets, is itself neither new or controversial. Indeed, it is an article of conventional wisdom embraced by rationalists and behavioralists alike. However the full force of the arbitrage principle in both justifying and tempering the quantification of economic rationality seems to have been underappreciated. The purpose of this paper is to demonstrate the scope of this principle in diverse contexts ranging from personal decisions to behavior in games and markets.

In all of these settings, money will play a central role not only as a means of facilitating transfers of commodities and strategically decoupling the actions of different agents, but also as the medium of communication through which they articulate their beliefs and values to each other - and even to themselves. Knowledge - and especially common knowledge - of an agent's beliefs and values will be signified by her willingness to engage in transactions in which goods (which may be distinct commodities or merely lottery tickets) are bought or sold at declared prices. This view of communication, in which the agent is expected to put her money where her mouth is, is admittedly narrow and stylized. Nonetheless, we argue that money becomes an indispensable yardstick at precisely the point where numbers must be attached to beliefs and values in a credible and operationally meaningful way. Monetary systems are ubiquitous in human culture, and the framing of judgments in terms of acceptable prices is a familiar cognitive activity to most real economic agents. Prices are also typically additive - at least for small transactions - and this property not only simplifies decision-making but also can be exploited to great advantage in the construction of analytical models.

The use of money as the medium of communication has the nontrivial side effect that transfers of information may be accompanied by transfers of wealth, giving rise to market interactions between an agent and her observers. Throughout the analysis presented here, it will be assumed that such markets clear. This will be seen to have implications for the inseparability of probabilities from utilities, and for the homogeneity of revealed probabilities, which have been pointed out by Kadane and Winkler (1988). Unlike those authors, we do not view these effects as problematic. Rather, we argue that only in a cleared market are we on firm ground in asserting that numerical probabilities 
and utilities are known (however imprecisely) to the agents, let alone that they are common knowledge. Although traditional subjective expected utility theory aims at prescribing behavior in arbitrary (e.g., non-equilibrium or non-market) situations, the detailed measurements needed to carry out such prescriptions are generally not obtainable.

The next section of the paper introduces the basic duality theorem connecting the arbitrage principle with the existence of subjective probability distributions or, more generally, "risk-neutral" distributions incorporating both probabilities and marginal utilities. Section 3 discusses the separability of beliefs and risk preferences, and Section 4 considers the question of completeness, at both the individual and market levels. Sections 5, 6, 7, and 8 consider applications of the arbitrage principle in decision analysis, securities markets, exchange economies, and noncooperative games.

\section{De FINETTI'S THEOREM AND RISK-NEUTRAL PROBABILITIES}

In a paper which strongly influenced Savage and other Bayesian statisticians, de Finetti (1937) proposed an operational definition of probability in terms of betting rates or prices placed on lottery tickets. Let $p(\mathbf{E})$ denote the unit price at which you would indifferently buy or sell lottery tickets paying $\$ 1$ if the event $\mathbf{E}$ occurs, and $\$ 0$ otherwise. Similarly, let $p(\mathbf{E} \mid \mathbf{F})$ denote the unit price at which you would buy or sell tickets paying $\$ 1$ if the events $\mathbf{E}$ and $\mathbf{F}$ both occur, $\$ 0$ if $\mathbf{F}$ occurs without $\mathbf{E}$, and a refund of the purchase price (i.e., a "called off bet") if $\mathbf{F}$ fails to occur. De Finetti showed that such a system of prices is coherent-i.e., does not adnit arbitrage - if and only if it fulfills the requirements of a probability measure. In particular, the prices must satisfy $p(\mathbf{E}) \geqslant 0, p(\mathbf{E})+p\left(\mathbf{E}^{\mathbf{c}}\right)=\mathbf{1}, p(\mathbf{E} \cup \mathbf{F})=p(\mathbf{E})+p(\mathbf{F})$ if $\mathbf{E} \cap \mathbf{F}=$ $\emptyset$, and $p(\mathbf{E} \cap \mathbf{F})=p(\mathbf{E} \mid \mathbf{F}) p(\mathbf{F})$. On this basis, de Finetti proposed that the prices should be interpreted as your probabilities and that the concept of probability has no other meaning beyond this. ${ }^{1}$

De Finetti gave a separate proof of each probability law based on the existence of a solution to a system of linear equations. It was later realized these results were a special case (indeed, an exquisite illustration) of the duality theoreni of linear programming. The duality between a coherent gambling system and the existence of a supporting 
probability measure is commonly referred to as "de Finetti's theorem", 2 and can be formalized in the following way:

DEFINITIONS. Let $\Theta$ denote a finite set of states of nature, exactly one of which will occur. A gamble is a vector whose elements, indexed by states of nature, represent monetary payoffs assigned to states. If $\mathbf{w}$ denotes an agent's current distribution of state-contingent wealth, then $\mathbf{x}$ is an acceptable gamble for her if she weakly prefers $\mathbf{w}+\alpha \mathbf{x}$ to $\mathbf{w}$ for any small ${ }^{3}$ positive $\alpha$ chosen "at the discretion of an opponent."

In other words, if an agent asserts that $\mathbf{x}$ is acceptable to her, then an opponent may choose a small positive number $\alpha$, and a contract will then be enforced in which there is a net payment of $\alpha x(\theta)$ from the opponent to the agent when state $\theta \in \Theta$ occurs. This is a gain for the agent and a loss for the opponent if $x(\theta)$ is positive, and vice versa if $x(\theta)$ is negative. For example, if $\$ p$ is a price at which the agent will indifferently buy or sell a lottery ticket paying $\$ 1$ if event $\mathbf{E}$ occurs, this means that the gambles whose payoff vectors are $\mathbf{E}-p$ and $p-\mathbf{E}$ are both acceptable. ${ }^{4}$ Unlike de Finetti, we will not generally assume the existence of a single price at which an agent indifferently buys or sells: she may wish to buy at a price no greater than $p$ and sell at no less than $q$, where $p<q$. That is, we allow the possibility of distinct lower and upper probabilities for events (Smith 1961).

If $\mathbf{x}$ is acceptable, it follows from the definition that $\alpha \mathbf{x}$ is also acceptable for sufficiently small $\alpha$-i.e. acceptable gambles are linearly divisible. Furthermore, we make the following:

ASSUMPTION (ADDITIVITY). If $\mathbf{x}$ and $\mathbf{y}$ are acceptable, then $\mathbf{x}+\mathbf{y}$ is acceptable.

In other words, the purchase or sale of one lottery ticket does not affect preferences with respect to other lottery tickets, if only because transactions are considered to be "small". Thus, for example, if an agent is willing to buy lottery tickets on disjoint events $\mathbf{E}_{1}$ and $\mathbf{E}_{2}$ at prices $p_{1}$ and $p_{2}$, then she must also buy lottery tickets on their union $\mathbf{E}_{1} \cup \mathbf{E}_{2}$ at price $p_{1}+p_{2}$. The additivity of prices is, as de Finetti (1974, p. 74) observes, "the foundation of the whole treatment" of subjective probability, since it allows linear duality theory to be invoked. 
THEOREM 1. Let $\mathbf{x}_{k}, k=1,2, \ldots$, denote acceptable gambles. Then there is no acceptable gamble with a strictly negative payoff (an arbitrage opportunity for an opponent) if and only if there exists a probability distribution $\pi$ on $\Theta$ such that $\pi^{\mathrm{T}} \mathbf{x}_{k} \geqslant 0$ for all $k-i . e ., a$ distribution assigning non-negative expected value to every acceptable gamble.

Proof: Let $X$ denote the matrix whose $k$ th column is $\mathbf{x}_{k}$. Then, by the linearity and additivity properties, $X \boldsymbol{\alpha}$ is an acceptable gamble for any small, non-negative vector $\boldsymbol{\alpha}$. By a separating-hyperplane theorem of linear algebra (Gale 1960, Theorem 2.10, a variant of Farkas' lemma), either the system of equations $X \boldsymbol{\alpha}<0$ has a non-negative solution (i.e., there is an acceptable gamble whose payoff is uniformly negative) or else the system $\pi^{\mathrm{T}} X \geqslant 0$ has a semi-positive solution, which w.l.o.g. can be scaled as a probability distribution.

The preceding theorem establishes that the coherent agent is one whose acceptance of gambles appears to be predicated on calculations of expected value with respect to some probability distribution over states. Under such a distribution, the prices at which she will indifferently buy or sell lottery tickets are just the probabilities of the corresponding events.

Although no-arbitrage (a.k.a. "coherence" or "admissibility" or "Dutch book") arguments based on de Finetti's theorem were used by Bayesian statisticians in the 1960's and 1970's to demonstrate the illogicality of frequentist statistical methods (e.g., Freedman and Purves 1969, Cornfield 1969, Lindley 1972, Heath and Sudderth 1972, Pierce 1973), his operational concept of probability was largely overshadowed by Savage's (1954) joint axiomatization of subjective probability and expected utility in terms of binary preferences. The lack of provision for a possibly-nonlinear utility function was portrayed by de Finetti himself as an apparent "limitation" of a theory of subjective uncertainty based on money bets (1937, footnote (a); 1974 pp. 77-81), and the (mis)conception that Dutch book arguments have limited economic realism still persists. For example, Yaari (1985) argues that, in the context of decision-making under risk, the avoidance of Dutch books requires risk-neutral behavior. Yaari's argument hinges on an implicit additivity-of-preference assumption, namely that "preference 
patterns... remain in force" whenever a more-preferred gamble is exchanged for a less-preferred one. However, this additivity assumption is reasonable only for gambles which are so small that they yield changes in wealth insufficient to affect other preferences. The appropriate conclusion is that the avoidance of Dutch books requires the appearance of risk-neutrality with respect to some distribution (not necessarily one's "true" distribution, as will be seen) when gambles are sufficiently small. This is not inconsistent with risk-averse or riskseeking behavior in-the-large, provided that preferences are smooth.

Notwithstanding its peripheral status in subjective expected utility theory, de Finetti's theorem surfaced again under different aliases in the explosion of "arbitrage pricing" literature in finance in the 1970's: securities markets epitomize the gambling environment envisioned by de Finetti for the elicitation of personal probabilities, and there the avoidance of arbitrage opportunities is a tangible concern. For example, consider the two-period asset pricing problem in which risky assets (securities) are bought and sold at time 0 and yield uncertain returns at time 1 . Let $\Theta$ denote the set of all possible joint returns, let $\mathbf{z}_{k}$ denote the vector of returns for one share of security $k$, and suppose that a representative agent is willing to buy or sell shares of security $k$ at prices $p_{k}$ and $q_{k}$, respectively, where $p_{k} \leqslant q_{k}{ }^{5}$ Let $r$ denote 1 plus the risk-free interest rate between periods, meaning that riskless bonds paying $\$ 1$ with certainty in period 2 can be bought or sold at price $\$ r^{-1}$ in period 1 . For simplicity, assume that the buying or selling prices for riskless bonds - i.e., borrowing and lending rates - are the same.

Under the preceding assumptions, a share of security $k$ can effectively be bought [sold] with a net payment [receipt] of $p_{k} r\left[q_{k} r\right]$ at period 2 , and the "gambles" whose period-2 payoff vectors are $\mathbf{z}_{k}-p_{k} r$ and $q_{k} r-\mathbf{z}_{k}$ are therefore "acceptable" to the agent in the terminology of Theorem 1. By application of the theorem, it follows that no arbitrage opportunities exist if and only if there exists a distribution $\pi$ such that $\boldsymbol{\pi}^{\mathrm{T}}\left(\mathbf{z}_{k}-p_{k} r\right) \geqslant 0$ and $\boldsymbol{\pi}^{\mathrm{T}}\left(q_{k} r-\mathbf{z}_{k}\right) \geqslant 0, \quad$ or equivalently $p_{k} \leqslant$ $r^{-1} \pi^{\mathrm{T}} \mathbf{z}_{k} \leqslant q_{k}$, for every $k$. In other words, there must exist a distribution under which every security's expected value, discounted at the riskless rate, lies between its advertised buying and selling prices. Such a distribution is known as a risk neutral probability distribution, since a risk-neutral agent with these probabilities would judge the 
securities to be fairly priced. The derivation of such "risk neutral valuation relationships" (a.k.a., linear valuation, state pricing, equivalent martingale measures) from no-arbitrage conditions via duality theorems has emerged as one of the most important tools in the modern theory of financial markets. ${ }^{6}$

An interesting feature of risk-neutral valuation is that in a complete and frictionless market (where $p_{k}=q_{k}$ for all $k$ ) every asset must appear to yield the same expected return per dollar invested, namely the riskless rate $r$. At first glance this seems to contradict the notion of a risk-return tradeoff, the very hallmark of prudent investment, and this has led to the somewhat misleading characterization of such results as "preference free" (Cox and Ross 1976). The contradiction disappears if $\pi$ is interpreted not as the "true" distribution of the representative agent, but as the (renormalized) product of her true probabilities and marginal utilities (Beja 1967). That is, we interpret $\pi$ as:

$$
\pi(\theta) \propto p(\theta) u^{\prime}(w(\theta))
$$

where $p(\theta)$ is the agent's true subjective probability for state $\theta, w(\theta)$ is her wealth in state $\theta, u(w)$ is her utility for wealth $w$, and $u^{\prime}(w)$ is her marginal utility for wealth evaluated at $w$. In this way, $\pi$ encodes the agent's risk preferences as well as her beliefs.

\section{THE INSEPARABILITY OF BELIEFS AND RISK PREFERENCES}

It has recently been shown by Kadane and Winkler (1988) that virtually any transactional method of eliciting probabilities (e.g., lotteries, scoring rules, promissory notes) will yield risk-neutral probabilities ( $\pi$ 's) instead of "true" probabilities ( $p$ 's) if the agent's wealth is correlated with the events under consideration, due to the confounding effect of state-dependent marginal utility. As they point out, the events for which an individual's probabilities are of interest - to herself or to others - are often precisely those in which she has a significant prior stake, either financially or reputationally. The implication is that we cannot separate personal probabilities from utilities without independently knowing both the individual's utility function and her 
current wealth distribution, which is generally impractical if not impossible.

This is not all: if the agent is embedded in an economy with complete markets for contingent claims, then market-clearing requires her risk-neutral probabilities to agree with those of all other agents! An efficient allocation of state-dependent wealth is not achieved until the renormalized product of every agent's probabilities and marginal utilities is the same. This fact, which follows by application of the arbitrage principle at the market level, will be discussed more fully in the next section. It was originally exploited by Wilson (1968) to derive Pareto optimal sharing rules for syndicates, and was also discussed by Drèze (1970, based on a 1965 lecture), before its emergence in the arbitrage literature of finance. Kadane and Winkler refer to this as a "startling and unreasonable" result, since it means that agents" "true" probabilities will be especially difficult to elicit in a market context, being confounded not only with their own marginal utilities but also with the probabilities and utilities of other agents.

We take a somewhat less pessimistic view, namely that the epistemic separation of beliefs from preferences, while useful from a conceptual standpoint, is inessential to the characterization of economic rationality in terms of observable behavior. A cleared market is the situation in which we are on firmest ground in quantifying any aspect of an agent's beliefs and preferences, since it is here that they have been most unequivocally translated into action. In the models discussed in later sections of this paper, it will be shown that through the very act of hedging herself against the market, an agent reveals everything which it is necessary to know about her personal beliefs and preferences. The market thereby serves as the fundamental instrument of reciprocal measurement among agents, rather than an obscuring influence. The fact that what we observe in the market are an agent's risk-neutral probabilities rather than her "true" probabilities and utilities, and that these may even be shared by other agents, is neither unnatural nor problematic. On the contrary, the interpretation of risk-neutral probabilities as an amalgam of beliefs and risk preferences is precisely what frees de Finetti's theorem from the apparent limitation of requiring "rigidity in the face of risk" (1974, p. 77), making it a completely general foundation for a theory of rational choice under uncertainty. 
An analogous problem of measurement is found in classical thermodynamics, where the fundamental system variable, namely entropy, is not directly observable. Instead, the observable variables include the derivative of entropy with respect to energy, which is the reciprocal temperature. A composite system is in thermal equilibrium if the temperature is constant across its components, and this is the situation in which infinitesimal measurements of temperature and other system variables are most reliable. Indeed, classical thermodynamics is mainly concerned with describing the net change from one equilibrium state to another as constraints are removed or parameters varied; an axiomatic treatment is given by Callen (1960).

In economic systems, expected utility plays the role of entropy, and state-dependent wealth plays the role of energy. For example, both entropy and expected utility are postulated to seek the maximum value allowed by constraints on the system; and expected utility is a monotonically increasing function of wealth, as is entropy with respect to energy. In welfare economics and group decision theory, expected utility is often considered to be, like entropy, additive across "subsystems" (agents); and wealth is usually assumed to be, like energy, a conserved quantity. The derivative of an agent's expected utility with respect to her wealth in state $\theta^{*}$ is:

$$
\frac{\partial}{\partial w\left(\theta^{*}\right)} \sum_{\theta} p(\theta) u(w(\theta))=p\left(\theta^{*}\right) u^{\prime}\left(w\left(\theta^{*}\right)\right)
$$

which is [proportional to] her risk-neutral probability for $\theta^{*}$, and is the analog of a reciprocal temperature in that state. In the absence of barriers to trade, wealth (energy) in each state will tend to flow from agents with low risk-neutral probabilities (high temperature) to agents with high risk-neutral probabilities (low temperature), until an equilibrium is reached in which the risk-neutral probabilities of all agents have been equalized. The analogy is of course imperfect - for example, the entropy-increase postulate must be enforced at the agent level, not just the system level - yet the formal similarities are strong enough to suggest that the difficulty of directly measuring "true" probabilities and utilities is no more problematic than the difficulty of directly measuring entropy. 


\section{COMPLETENESS AND THE REPRESENTATIVE AGENT}

Thus far is has been shown that rational (coherent) behavior under uncertainty requires the existence of a supporting risk-neutral probability distribution under which every acceptable transaction has nonnegative expected value. If the additional assumption is made that beliefs and preferences are completely ordered -i.e., that between any two alternatives an agent can always assert a direction of weak preference - then it follows that the risk-neutral distribution must be unique. (In this case, for every state $j$, there must exist a unique price above which the agent is willing to sell a lottery ticket on $j$ and below which she is willing to buy it, which is the unique risk-neutral probability of state $j$.) The completeness assumption is commonly invoked in normative decision theory, but it has also been emphatically rejected by a long line of researchers extending from Keynes (1921) to Walley (1991), ${ }^{7}$ and it is the latter position which we endorse here. Completeness is certainly useful as a technical assumption if it is desired to obtain deterministic and analytically convenient representations of agent behavior in special cases (examples of which will be given in later sections), but it is inessential to the general characterization of rationality provided by the arbitrage principle.

An agent's greatest buying price and least selling price for a lottery ticket impose linear constraints on her set of supporting risk neutral distributions, and the intersection of these constraints is a convex set. This is illustrated in Figure 1 for the case of a 3-element state space. The set of all risk-neutral distributions is the simplex of non-negative $\pi=\left(\pi_{1}, \pi_{2}, \pi_{3}\right)$ satisfying $\pi_{1}+\pi_{2}+\pi_{3}=1$, which is shown in 2 dimensional space with coordinates $\left(\pi_{1}, \pi_{2}\right)$, and $\pi_{3} \equiv 1-\pi_{1}-\pi_{2}$. Let $p_{j}$ and $q_{j}$ denote the agent's greatest buying and least selling prices, respectively, for a lottery ticket paying $\$ 1$ if state $j$ occurs, for $j=1,2$. In other words, $p_{j}$ and $q_{j}$ are her lower and upper risk-neutral probabilities for state $j$. The set of risk-neutral distributions consistent with these prices is the set of all $\pi$ satisfying $p_{j} \leqslant \pi_{j} \leqslant q_{j}$ for $j=1,2$, which is the intersection of the shaded bands in Figure 1. If the agent also assesses buying and selling prices on "mutual funds" composed of weighted sums of lottery tickets on different states, her set of riskneutral distributions may be constrained even further-say, to the 


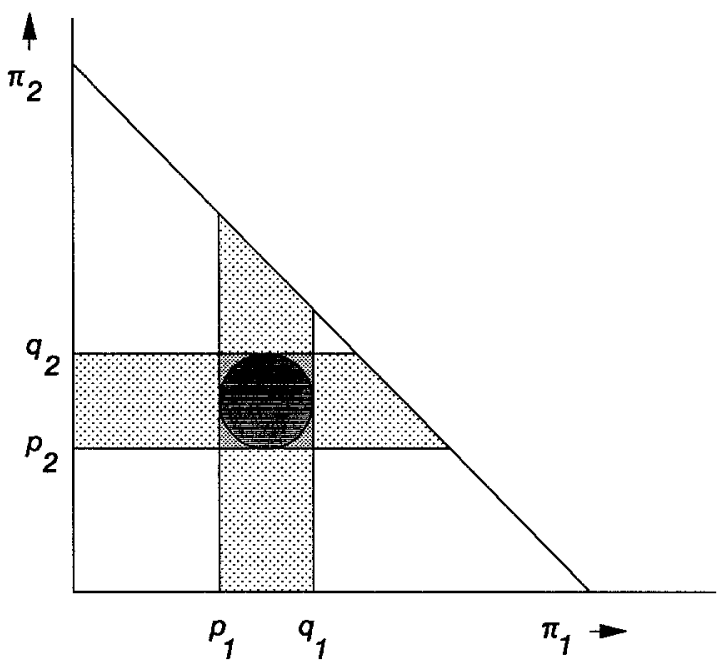

Fig. 1. Set of risk-neutral distributions revealed by an incomplete agent's buying and selling prices for lottery tickets on states 1 and 2 .

darkly shaded circular region in the center. If the completeness assumption holds - i.e., if the agent's greatest buying and least selling prices always coincide - her set of risk-neutral distributions reduces to a unique point in the simplex. In the absence of completeness, coherence merely requires that her set of risk-neutral distributions should be non-empty.

In a market with many agents, the additivity property of acceptable transactions naturally extends to inter-agent as well as intra-agent behavior, giving rise to the appearance of a "representative" agent. For example, if agent 1 is willing to buy a lottery ticket on an event $\mathbf{E}_{1}$ at price $p_{1}$, and agent 2 is willing to buy a ticket on a disjoint event $\mathbf{E}_{2}$ at price $p_{2}$, then from the perspective of a third party it is possible to sell a ticket on $\mathbf{E}_{1} \cup \mathbf{E}_{2}$ at price $p_{1}+p_{2}$ to the representative agent. In general, the set of acceptable gambles for the representative agent is the sum of the sets of acceptable gambles for the real agents. Dually, the representative agent's set of risk-neutral distributions is the intersection of the sets of risk-neutral distributions of the real agents, and by Theorem 1 the market admits no arbitrage opportunities if and only if this intersection is non-empty. In this case, the market is in 
equilibrium, since every agent's preferences are in accord with market prices.

For example, the representative agent's selling price for a lottery ticket on state $j$ is no greater than the minimum selling price among the real agents, and may even be strictly smaller if a lottery ticket on state $j$ can be constructed out of a portfolio of mutual funds sold to different agents. This is illustrated in Figure 2 for the case of three agents, whose sets of risk-neutral probabilities are labeled $A, B$, and $C$. The representative agent's buying and selling prices for lottery tickets on state $j$ are shown as $p_{j}^{*}$ and $q_{i}^{*}$ for $j=1,2$. In this illustration, the beliefs and preferences of the real agents are incomplete, and so are those of the representative agent, although the latter are "more complete" than the former in the sense that they yield narrower spreads between buying and selling prices.

Figure 3 illustrates the situation in which the beliefs and preferences of the representative agent are complete, despite the fact that those of the real agents are not: the representative agent's risk-neutral distribution is uniquely determined. This situation might obtain in the presence of genuine vagueness in agent beliefs and preferences pro-

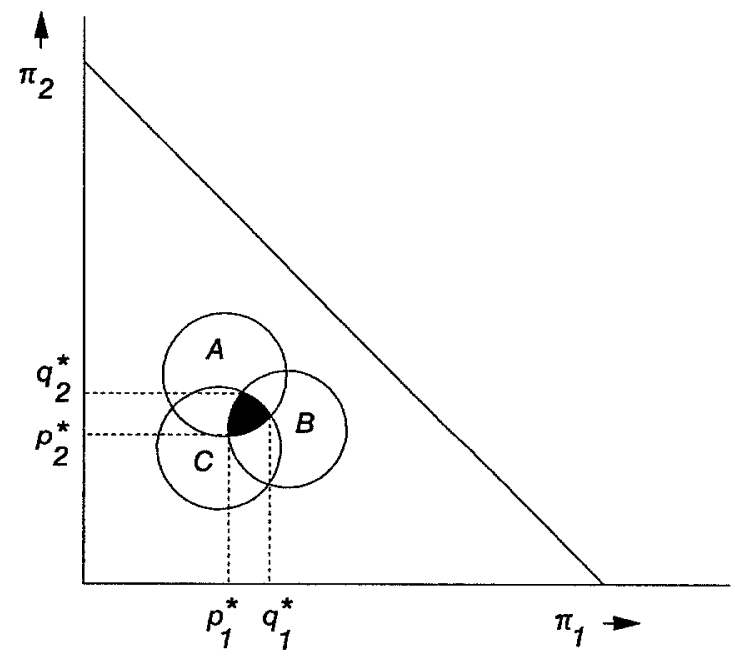

Fig. 2. Set of risk-neutral distributions of an incomplete representative agent composed of three incomplete real agents. 


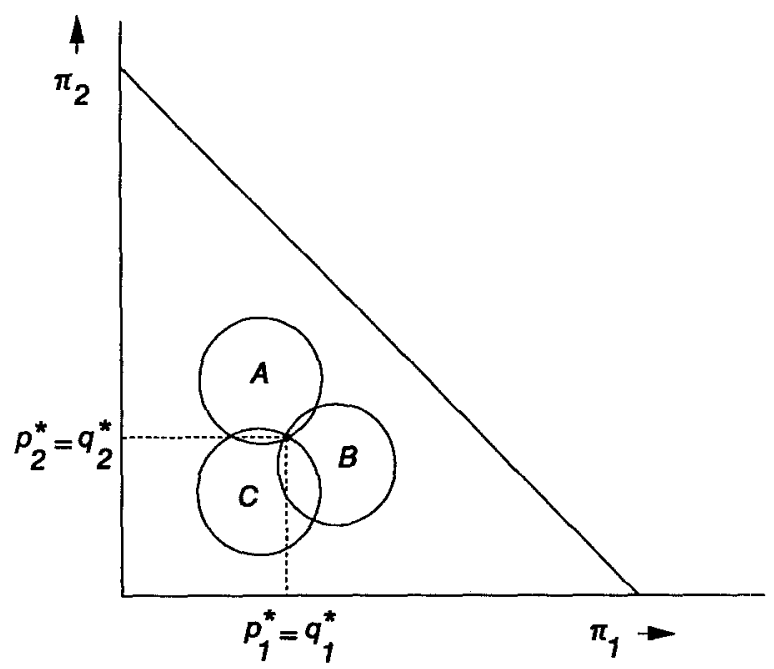

Fig. 3. Unique risk-neutral distribution of a complete representative agent composed of three incomplete real agents.

vided that they were sufficiently heterogeneous and the market sufficiently frictionless.

Figure 4 illustrates the situation in which every real agent's beliefs and preferences are complete, so that each has a unique risk-neutral distribution. The market no-arbitrage condition is that these must all coincide, in which case the representative agent is indistinguishable from any of the real agents. However, this homogeneity of risk neutral distributions does not necessarily imply homogeneity of "true" beliefs: the agents may have different true probabilities as long as the (renormalized) products of these with their marginal utilities yield the same result. For example, we might imagine that the agents originally held different risk neutral distributions in the primordial disequilibrium state before the market was opened. Subsequently, through the trading of securities, they would have redistributed wealth or information among themselves until the differences were erased. Thus, agents with greater risk tolerance presumably would have ended up holding more risky portfolios, those who judged a certain state more likely would have bet more of their wealth on it, and so on. The discovery that their apparent probabilities were initially different, giving rise to trade, 


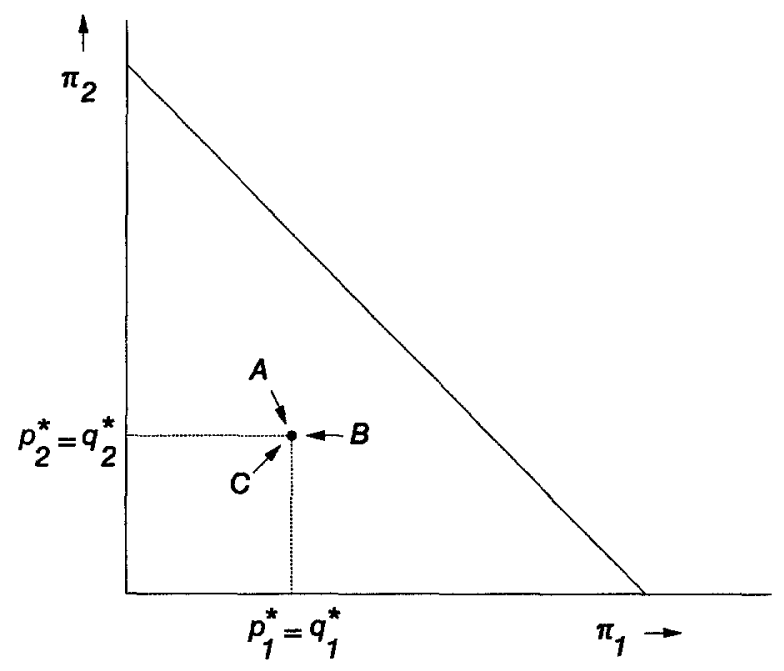

Fig. 4. Unique risk-neutral distribution of a complete representative agent composed of three complete real agents.

might have led to Bayesian updating as well as redistribution of wealth. However, as noted earlier, it would generally be difficult if not impossible for one agent to determine the extent to which changes in another's apparent probabilities - or her own - were due to acquisition of information, redistribution of wealth, or reassessed attitude toward risk: learning would be indistinguishable from hedging.

The incomplete-markets situation depicted in Figure 2 should clearly be regarded as the norm, if only because of friction (transaction costs) and the impracticability of maintaining liquid markets in enough linearly independent securities to span the high-dimensional (indeed, infinite dimensional) state spaces encountered in practice. However, the dominant effect may be genuine incompleteness in the belief- and preference-orderings of individual agents: if individual beliefs and preferences were truly complete, every chance meeting between two different individuals would give rise to a flurry of barter and gambling, a securities market in miniature. The fact that individuals typically exhibit inertia and aversion to low-stakes gambles is strong evidence of incompleteness ("internal friction"). Indeed, real investment and consumption decisions are usually predicated not on exacting calculations 
of personal expected utility, but rather on habits, traditions, rules of thumb, solicitation, peer pressure - or impersonal attempts to exploit market inefficiencies (e.g., program trading in capital markets). The similarity of security price movements to Brownian motion is therefore not coincidental: prices are bumped upwards or downwards by unpredictable collisions with shifting cliques of agents, who are subject to untold personal influences. As suggested by Figures 2 and 3, the buying and selling prices of different securities will generally be influenced by different subsets of investors.

Agents are not endowed at birth with probabilities, utilities, and computing machinery sufficient to determine a program of optimal lifetime investment and consumption, subject only to Bayesian updating upon the receipt of new information. Rather, they gradually acquire decision-making rules and other norms of economic behavior through imitation and adaptation, and then contribute modestly to their propagation and mutation. Yet, the argument is often made that this evolutionary process will eventually produce complete and stable preference orderings at the individual level - at least in competitive markets - and on this basis the market is analyzed from the "bottom up" by explicitly solving the intertemporal optimization problem of the complete agent. The examples in this section are intended to make the case for a "top down" approach. The principles which justify the quantification of rationality at the individual level (linearity, additivity, and no arbitrage opportunities) are the same principles which apply even more forcefully at the market level, so that the market is generally more complete in its rationality than any of the individuals it comprises. Each real agent is (by definition) only an incomplete image of the representative agent, who in turn may be only an incomplete image of homo aconomicus rationalis.

\section{ARBITRAGE AND EQUILIBRIUM IN DECISION ANALYSIS}

In the remainder of the paper, we survey the application of arbitrage arguments and risk-neutral probabilities (or related kinds of dual variables) in different domains of economic modeling. We begin with decision analysis, where the "old time religion" of subjective expected utility theory finds its most direct application. In particular, we con- 
sider the analysis of decisions which are made in a market context (e.g., capital budgeting decisions), where risk-neutral probabilities for the relevant events might be obtainable from observed prices of securities, commodities, and contingent claims. In the corporate finance literature, it has been asserted (e.g., by Banz and Miller 1978 and Trigeorgis and Mason 1987) that "contingent claims analysis" based on risk-neutral probabilities is superior to conventional discounted cash flow analysis or decision tree analysis in this context. In this section we examine the basis of this claim and consider some of the issues it raises. (A more thorough treatment of arbitrage in decision analysis is given by Nau 1991b.)

As an example consider the capital budgeting problem whose decision tree is shown in Figure 5. (This is adapted from an example given by Trigeorgis and Mason and also used by Copeland et al. 1990.) The corporation has an opportunity to invest in a new plant whose returns depend on an uncertain state of the world, which will be "good" with probability $p$ or "bad" with probability $1-p$ (as assessed by the decision maker). If $\$ P$ is invested in year 1 , the returns in year 2 will be $\$ 180$ in a good state or $\$ 60$ in a bad state. Alternatively, the

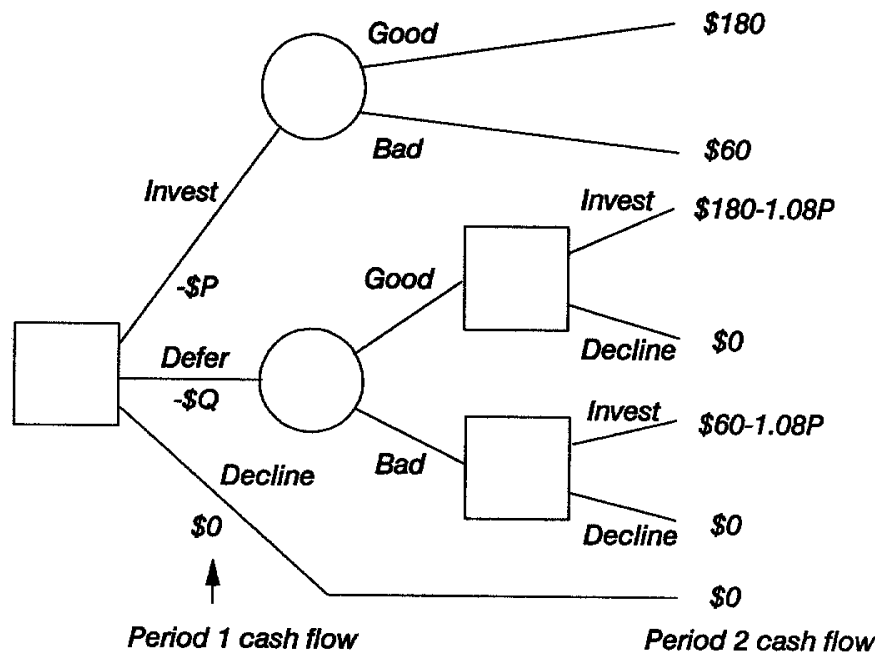

Fig. 5. Decision tree for capital budgeting problem. 
company can pay $\$ Q$ in year 1 for a license which allows them to defer making the investment decision until the state of nature becomes known. If they choose this option, then in year 2 they can invest $\$ 1.08 P$ and reap a known return ( $\$ 180$ or $\$ 60)$, or else decline to invest, where the factor of 1.08 reflects a risk-free interest rate of $8 \%$. The status quo (decline immediately, for a current and future payoff of $\$ 0)$ is also an option.

A naive decision tree analysis based on discounted cash flows would begin with the selection of a discount rate, intended to reflect both the time value of money and a premium for risk. The decision with the highest expected net present value would then be selected. An appropriate discount rate might simply be dictated by company policy, or it might be derived from market prices using a CAPM-type approach. In the latter regard, suppose that there exists a "twin security" whose returns are believed to be perfectly correlated with those of the plant in question: the value of the twin security in a good state will be, like that of the plant, three times its value in a bad state. (For example, the plant's return may depend on interest rates or prices of commodities for which active futures and options markets exist.) For concreteness, suppose that one share of this security will be worth $\$ 36$ in a good state and $\$ 12$ in a bad state, whereas its current price is $\$ S$. If $p$ is considered the "true" probability of a good state, the market rate of return on this security, $r$, satisfies $r=(36(p)+12(1-p)) / S=$ $(24 p+12) / S$. For example, if $S=20$ and $p=0.5$, we obtain $r=1.2$ : the message of the market is that a project with this kind of risk should earn a $20 \%$ return. Using this implied rate of return, the option of investing immediately has an expected net present value of $\$(180(p)+60(1-p)) / r-P=\$ 5 S-P=\$ 100-P$. If $P=104$, for example, then investing immediately yields an expected NPV of $\$ 100-$ $104=-\$ 4$; this is inferior to the status quo, which of course has an NPV of $\$ 0$. Note that the derived rate of return, $r$, depends on the value assumed for the probability of a good state, $p$. However, in the final calculation, the contributions of $p$ and $r$ cancel between numerator and denominator: the present value of the returns on the plant is simply proportional to the price of the twin security.

Now consider the evaluation of the "defer" option, and suppose, as before, that $p=0.5, S=20$ (whence $r=1.2$ ), and $P=104$. For what 
values of $Q$ will this option be preferred to the status quo? The naive decision tree analysis would observe that the optimal deferred decision is "invest" in a good state (for a year-2 return of $\$ 180-(1.08) 104=$ $\$ 67.68$ ) and "decline" in a bad state (for a year-2 return of $\$ 0$ ); discounting these values back to the present at $20 \%$ yields an expected NPV of $\$((67.68(0.5)+0(0.5)) / 1.2)-Q=\$ 28.20-Q$. Hence the "defer" option should be chosen if $Q<28.20$.

In contrast, the contingent-claims-analysis approach would discount the year- 2 cash flows at the risk-free rate (here assumed to be $8 \%$ ), while taking expectations with respect to risk-neutral probabilities inferred from market prices. Thus, the premium for risk would be impounded with the probability distribution rather than with the discount rate. In this example, the risk-neutral probability of a good state, denoted $\pi$, is obtained by equating the price of the twin security with its expected return discounted at the risk-free rate: $\$ 20=$ $\$(36(\pi)+12(1-\pi)) / 1.08$, whence $\pi=0.4$. This leads to the same evaluation as the naive approach for the "invest immediately" option: its expected net present value is $\$(180(0.4)+60(0.6))$ / $1.08-P=\$ 100-P$, as before. However, for the "defer" option, using $P=104$, we now obtain an expected net present value of $\$(67.68(0.4)+0(0.6)) / 1.08-Q=\$ 25.07-Q$. Hence, this option has a positive expected net present value (and is superior to the status quo) only if $Q<25.07$. Another way to obtain this last result is to go back to the arbitrage argument underlying the derivation of risk-neutral probabilities: by solving a system of two equations in two unknowns, it can be determined that buying 2.82 shares of the twin security and selling $\$ 31.33$ worth of risk-free $8 \%$ bonds will exactly replicate the year-2 payoffs of the "defer" option, and the year-1 cost of this transaction is $2.82(\$ 20)-\$ 31.33=\$ 25.07$. Hence, setting a value on the "defer" option other than $\$ 25.07$ would violate the law of a single price, giving rise to profitable arbitrage opportunities.

On the basis of examples such as this, it has been claimed that naive decision tree analysis using discounted cash flows tends to misjudge the value of "managerial flexibility" in sequential decision-making contexts. It would be more accurate to say that the naive approach does not properly consider risk preferences (i.e., the decision maker's utilities), and hence overlooks the opportunities for hedging which are 
implicitly considered in the contingent claims approach. The validity of the contingent claims approach rests on the assumption that the decision-maker can adopt the market's risk-neutral probabilities as her own. This, in turn, assumes that the decisions-maker maintains an equilibrium between herself and the market by augmenting her investment decisions with transactions in marketed securities in such a way that her own risk-neutral probability distribution coincides with that of the market.

A correct decision tree analysis of this situation would begin with the assessment of the decision-maker's utility function as well as her probability distribution - notwithstanding the caveats raised in the preceding sections about their determinacy or separability. The decision-maker's risk preferences would thus be separated both from her time preferences and her beliefs, and the augmented capitalbudgeting/hedging decision would be solved by maximizing her subjective expected utility. This would necessarily lead to the same valuations of the capital-budgeting options that were obtained by contingent claims analysis, but they would be accompanied by the explicit construction of hedged positions in the surrounding market.

For simplicity, suppose that no consumption will take place in year 1 , and that the decision maker's utility can be considered as a function of a single attribute, namely her net wealth in year $2 .{ }^{8}$ Suppose that her utility function for year- 2 wealth is exponential with an assessed risk tolerance of $\$ 200-$ i.e., $u(x) \propto 1-\exp (-x / 200)$. Given this information, together with the data above, it follows that in equilibrium the decision-maker should have some wealth invested in the twin security even under the status quo. A convenient property of the exponential utility function is that it exhibits constant absolute risk aversion (CARA), and hence the optimal investment decision is independent of the initial wealth level. Let $s$ denote the number of shares invested in the twin security. To determine the optimal value of $s$ under the status quo, we solve:

$$
\frac{\pi}{1-\pi}=\frac{p}{1-p} \frac{u^{\prime}((36-1.08(20)) s)}{u^{\prime}((12-1.08(20)) s)}
$$

where $u^{\prime}(x) \propto \exp (-x / 200)$ is the marginal utility of money at wealth 
level $x$. Plugging in $p=0.5$ and $\pi=0.4$, we obtain $2 / 3=\exp (-24 s /$ $200), s=-(200 / 24) \ln (2 / 3)=3.38$ shares. Note that the initial cost of the security investment, namely $\$ 20 s$, has been discounted forward at the risk-free rate of $8 \%$, as though the money to buy the security had been borrowed at $8 \%$ and repayed in year 2. Although this term subsequently drops out of the calculation because of the CARA property of the exponential utility function, it emphasizes that in principle the net values of decision options in year 2 are computed by discounting at the risk-free rate.

Now consider the option to invest in the new plant immediately, and note that the returns generated by the plant are exactly replicated by 5 shares of the twin security, which currently sells for $\$ 20$ per share. If the cost of this option $(\$ P)$ is greater than the cost of 5 shares of the twin security $(\$ 100)$, the option is obviously unattractive. On the other hand, if $P$ is less than $\$ 100$, this presents an arbitrage opportunity to the decision-maker relative to the status quo: she can invest $\$ P$ in the plant and sell 5 shares of the twin security for $\$ 100$, ending up with $3.38-5=-1.62$ shares (a "short" position), thereby keeping the same profile of year-2 payoffs while increasing her initial wealth. The fact that it is optimal for her to sell exactly 5 shares, so as to maintain precisely the same profile of year- 2 payoffs, is a consequence of the CARA property of the exponential utility function. Under a more general utility function, a more subtle hedging strategy would be called for.

Next, consider the option to defer the investment decision for an initial payment of $Q$, assuming that $P=104$. In year 2 , this option yields at most $\$ 67.68$ in the good state and $\$ 0$ in the bad state, a position which (as noted earlier) can be replicated by buying 2.82 shares of the twin security and selling $\$ 31.33$ worth of risk-free bonds paying $8 \%$, for a year- 1 cash outlay of $\$ 25.07$. Hence, if $Q>25.07$, the deferral option is less attractive than what is already available in the market, and the decision-maker will prefer to adhere to her status quo investment. If $Q<25.07$, she will perceive an arbitrage opportunity and take the deferral option while divesting herself of 2.82 shares of the twin security, ending up with $3.38-2.82=0.56$ shares, once again keeping her original profile of year- 2 payoffs while increasing her initial wealth. 
Several conclusions can be drawn from this example. One is that the problem with traditional decision tree analysis, as it is reportedly practiced in the corporate finance arena, is merely that it is practiced incorrectly: the decision-maker's risk preferences are erroneously confounded with time preferences, and secondary decision-making opportunities are consequently misvalued or overlooked. If these errors are corrected, decision tree analysis becomes equivalent to contingent claims analysis in problems for which the relevant markets exist. On the other hand, it can be argued that the contingent-claims approach provides a useful decomposition of the problem into two parts: (i) determining the primary decision which is optimal according to the market's risk-neutral valuation; and (ii) determining the secondary, portfolio-balancing decision which optimally hedges the primary decision with respect to the decision-maker's own beliefs and preferences. Under this decomposition, the act of solving (ii), by whatever means, replaces the a priori measurement of personal probabilities and utilities in conventional decision analysis. Contingent claims analysis explicitly confines itself to problem (i) while leaving problem (ii) up to unspecified and perhaps qualitative solution methods (executive judgment, rules of thumb, etc.). This may be advantageous if numerical probabilities and utilities are difficult to elicit or awkward to reveal for political and/or legalistic reasons. For example, whose utility should be maximized: the executive's, the corporation's, or the shareholders'? If the shareholders' perspective is adopted, it may be argued that they can and should balance their own portfolios, which is the rationale underlying the capital structure theorem of Modigliani and Miller.

Of course, perfectly correlated "twin securities" will generally not be available, although the existence of even imperfectly correlated securities would still enable contingent claims analysis to establish lower and upper bounds on the market valuation of decision options, and thereby provide some basis for hedging in a full-blown expectedutility analysis. However, many decision-making problems arise in settings where no relevant securities markets exist at all. In such cases, practitioners have found that the quantitative features of a decision model (i.e., the numerical expected utilities it produces) usually do not turn out to be pivotal: the greatest benefit is obtained from the qualitative process of structuring the problem, acquiring information, 
clarifying ambiguities, generating options, identifying critical variables, fostering communication, reconciling conflicting judgments, etc. ${ }^{9}$ This is not inconsistent with an evolutionary view of rationality: the formal analytic process serves to embed the primary decision problem in a richer context, in which the decision-maker's acquired heuristics augmented by those of the decision analyst - are more likely to find purchase. Of course, to the extent that formal decision analysis based on subjective expected utility theory is itself a useful heuristic, it should be expected to displace its normatively inferior competitors.

\section{ARBITRAGE AND EQUILIBRIUM IN A SECURITIES MARKET}

The example in the preceding section illustrates how the market acts as a kind of "heat sink," pulling the risk-neutral probabilities of an individual agent into line with those of the general population. In this section, we consider the question of how the market's risk-neutral probabilities are themselves formed by aggregation of the beliefs and risk preferences of its constituent agents - i.e., we consider the nature of a competitive equilibrium in a securities market. This analysis will also serve to highlight some of the issues raised earlier concerning the separability of probability, utility, and wealth. For analytic tractability, we consider a discrete-time, two-period economy with a finite set of primary securities and complete markets for contingent claims, under restrictive assumptions about the shape of individual probability distributions and utility functions. We put aside, for the moment, the question of completeness raised in Section 4.

It is well known (at least among Bayesians) that certain families of probability distributions are conjugate to each other with respect to Bayesian updating: if the likelihood function is conjugate to the prior distribution, then the corresponding posterior distribution belongs to the same family as the prior. A similar useful conjugacy exists between two important families of probability distributions, utility functions and wealth functions with respect to the calculation of risk-neutral probability distributions: if the agent's utility and wealth functions are conjugate to her true probability distribution, then her risk-neutral distribution belongs to the same family as her true distribution. These conjugacies have been explicitly discussed by Wilson (1968), Rubinstein (1976), Breeden and Litzenberger (1978), Brennan (1979), and 
Stapleton and Subrahmanyam (1984), and are implicitly at the root of many important closed-form results in financial economics. The conjugate relationship we shall exploit here is that which exists between the normal probability distribution, exponential utility function, and quadratic wealth function:

LEMMA. Consider an economy in which investments may be made in a finite number of securities in period 1 , yielding uncertain returns in period 2. Let the state of nature in period 2, denoted $\boldsymbol{\theta}$, be defined as the vector of returns per dollar invested in the primary securities, and suppose that:

(a) agent i's "true" probability density function for $\boldsymbol{\theta}$ is multivariate normal with mean vector $\boldsymbol{\mu}_{i}$ and covariance matrix $\$_{i}$ - that is:

$$
p_{i}(\boldsymbol{\theta}) \propto \exp \left(-\frac{1}{2}\left(\boldsymbol{\mu}_{i}-\boldsymbol{\theta}\right)^{\mathrm{T}} \Varangle_{i}^{-1}\left(\boldsymbol{\mu}_{i}-\boldsymbol{\theta}\right)\right) ;
$$

(b) her utility for terminal (period-2) wealth $w$ is exponential with risk tolerance $t_{i}$ - that is:

$$
u_{i}(w) \propto 1-\exp \left(-w / t_{i}\right), \quad \text { whence } u_{i}^{\prime}(w) \propto \exp \left(-w / t_{i}\right) ;
$$

and

(c) her terminal wealth is a quadratic function of $\boldsymbol{\theta}-$ that is:

$$
w_{i}(\boldsymbol{\theta})=a_{i}+\mathbf{b}_{i}^{\mathrm{T}} \boldsymbol{\theta}+\frac{1}{2} \boldsymbol{\theta}^{\mathrm{T}} C_{i} \boldsymbol{\theta} .
$$

(Here, $a_{i}$ is an amount of cash in period-2 dollars, $\mathbf{b}_{i}$ is a vector specifying the amount of money invested in each primary security, and $C_{i}$ represents holdings of nonlinear contingent claims - such as options - yielding a quadratic payoff in terms of the primary securities. The explicit use of quadratic wealth functions in this context is novel.) $)^{10}$

Then the agent's risk-neutral probability density, $\pi$, is multivariate normal with co-variance matrix $\$$ and mean $\boldsymbol{\mu}$, where:

$$
\begin{aligned}
& \ddagger=\left(\$_{i}^{-1}+C_{i} / t_{i}\right)^{-1} \text { and } \\
& \boldsymbol{\mu}=\$\left(\$_{i}^{-1} \boldsymbol{\mu}_{i}-\mathbf{b}_{i} / t_{i}\right) .
\end{aligned}
$$


The proof follows by substitution in (2.1). A similar conjugate relationship exists between the lognormal distribution, power (CRRA) utility function, and $\log$-log quadratic wealth function.

These formulae make explicit how the agent's risk tolerance and wealth distribution are impounded in the apparent probability distribution revealed by her marginal investment behavior. In particular, the quadratic term $\left(C_{i}\right)$ in the wealth distribution is directly associated with shifts in the apparent covariance, and the linear term $\left(\mathbf{b}_{i}\right)$ is directly associated with shifts in the apparent mean, while the risk tolerance parameter, $t_{i}$, serves as the natural unit of currency. Only the ratios of $\mathbf{b}_{i}$ and $C_{i}$ to $t_{i}$-i.e., the quantities invested in securities and contingent claims per unit of risk tolerance - are ultimately relevant. ( $\boldsymbol{\mu}_{i}$ and $\$_{i}$ are, like $\boldsymbol{\theta}$, unitless; $a_{i}, \mathbf{b}_{i}, C_{i}$, and $t_{i}$ are measured in units of money.)

Now consider the nature of an equilibrium in a two-period economy populated by agents with multivariate normal beliefs and exponential utility functions, as characterized by the lemma above. Since the beliefs and preferences of all agents are here assumed complete, equilibrium requires their risk-neutral probability distributions to be identical to the market risk-neutral distribution. (This is conceptually the same situation as depicted in Figure 4, although the state space here is infinite.) In addition to a finite number of risky securities, we assume that the market contains risk-free bonds, and let $r$ denote 1 plus the risk-free rate of return-i.e., $\$ r^{-1}$ is the (endogenously determined) price of a bond paying $\$ 1$ in period 2 . We continue to assume complete markets for contingent claims so that arbitrary wealth functions may be constructed and the market risk-neutral distribution may be revealed in arbitrary detail. ${ }^{11}$ It follows that the mean vector of the common risk-neutral distribution must be the vector whose elements are identically equal to $r$-i.e., the risk-neutral expected return per dollar invested in any security must equal the risk-free return, as noted in Section 2. Let $\boldsymbol{\mu}_{i}$ and $\$_{i}$ denote the mean and covariance of the "true" distribution of agent $i$, let $t_{i}$ denote her risk tolerance, and let her wealth function be $w_{i}(\boldsymbol{\theta})=a_{i}+\mathbf{b}_{i}^{\mathrm{T}} \boldsymbol{\theta}+\frac{1}{2} \boldsymbol{\theta}^{\mathrm{T}} C_{i} \boldsymbol{\theta}$ for some constant $a_{i}$, vector $\mathbf{b}_{i}$, and matrix $C_{i}$. Then, from the results of the lemma, the risk-neutral distributions of all agents coincide (i.e., an equilibrium obtains) if and only if: 


$$
\begin{aligned}
& \left(\ddagger_{i}^{-1}+C_{i} / t_{i}\right)^{-1}=\ddagger, \text { and } \\
& \$\left(\$_{i}^{-1} \boldsymbol{\mu}_{i}-\mathbf{b}_{i} / t_{i}\right)=\mathbf{r}
\end{aligned}
$$

for all $i$, where $\$$ denotes the observed covariance of the market risk-neutral distribution and $\mathbf{r}$ denotes its mean vector (whose elements are identically equal to $r$ ). By rearrangement, it follows that $C_{i}$ and $\mathbf{b}_{i}$ satisfy:

$$
\begin{gathered}
C_{i}=t_{i}\left(\ddagger^{-1}-\ddagger_{i}^{-1}\right), \\
\mathbf{b}_{i}=t_{i}\left(\$_{i}^{-1} \boldsymbol{\mu}_{i}-\ddagger^{-1} \mathbf{r}\right),
\end{gathered}
$$

or equivalently:

$$
\begin{aligned}
& \$_{i}=\left(\$^{-1}-C_{i} / t_{i}\right)^{-1}, \\
& \boldsymbol{\mu}_{i}=\$_{i}\left(\mathbf{t}^{-1} \mathbf{r}+\mathbf{b}_{i} / t_{i}\right) .
\end{aligned}
$$

Thus, under the assumptions of multivariate normal beliefs and constant absolute risk aversion, the mean and covariance of agent $i$ 's "true" distribution are revealed up to a scaling factor by agent $i$ 's holdings of securities: $\boldsymbol{\mu}_{i}$ and $\$_{i}$ can be computed from the observable quantities $r, \$, \mathbf{b}_{i}$, and $C_{i}$, but only if we independently know agent $i$ 's risk tolerance, $t_{i}$. (In particular, the term $C_{i} / t_{i}$ is the difference between agent $i$ 's inverse covariance matrix and that of the market.) Even in this highly idealized setting, in which complete preferences and observable wealth distributions have been assumed, agent $i$ 's beliefs are still confounded with her risk tolerance parameter.

It remains to show how the market's risk-neutral covariance, $\$$, is obtained by aggregating the "true" covariances of the agents. Toward this end, let $t \equiv \Sigma_{i} t_{i}$ denote the aggregate risk tolerance; let $\mathbf{b} \equiv \Sigma_{i} \mathbf{b}_{i}$ denote the aggregate vector of wealth invested in the primary securities; and let $\boldsymbol{\nu} \equiv \mathbf{b} / \Sigma_{i k} b_{i k}$ be the vector of weights describing the "market portfolio" - i.e., the proportions of aggregate wealth invested in different primary securities. (Here $b_{i k}$ denotes the $k t h$ element of $\mathbf{b}_{i}$, i.e., the amount of wealth invested by $i$ in security $k$.) Let $\boldsymbol{\theta} \equiv \boldsymbol{\nu}^{\mathrm{T}} \boldsymbol{\theta}$ 
denote the uncertain return on the market portfolio; and let $\sigma^{2} \equiv$ $\nu^{\mathrm{T}}+\nu$ denote the variance of the market portfolio derived from the risk-neutral co-variance matrix. Under these conditions, we obtain:

THEOREM 2. In an equilibrium with zero net holdings of contingent claims $\left(\Sigma_{i} C_{i}=0\right)$, the market's risk-neutral covariance is given by:

$$
\ddagger^{-1}=\sum_{i}\left(t_{i} / t\right) \$_{i}^{-1}
$$

i.e., the risk-neutral precision (inverse covariance) matrix is a weighted average of the agents' true precision matrices, with weights proportional to individual risk tolerances. Furthermore, if we define the aggregate vector of "true" mean stock returns as the vector weighted average: 12

$$
\boldsymbol{\mu} \equiv \sum_{i}\left(t_{i} / t\right) \$ \$_{i}^{-1} \mu_{i}
$$

then the aggregate expected return on the market portfolio is $\mu \equiv \nu^{\mathrm{T}} \boldsymbol{\mu}$, and it follows that the aggregate mean, covariance, risk tolerance, and wealth invested in stocks satisfy:

$$
\boldsymbol{\mu}-\boldsymbol{r}=\$ \mathbf{b} / t=\boldsymbol{\beta}(\mu-r)
$$

where:

$$
\boldsymbol{\beta}=\operatorname{Cov}(\boldsymbol{\theta}, \theta) / \boldsymbol{\sigma}^{2}=\frac{\boldsymbol{\nu}^{\mathrm{T}} \dot{ }}{\boldsymbol{\nu}^{\mathrm{T}} \dot{\psi} \boldsymbol{\nu}} .
$$

That is, $\beta_{k}$ (the kth element of $\boldsymbol{\beta}$ ) is the slope coefficient in the simple regression of security $k$ 's return $\left(\theta_{k}\right)$ on the market return $\left(\theta \equiv \nu^{\mathrm{T}} \boldsymbol{\theta}\right)$, based on the risk-neutral covariance matrix of the market.

Proof: (6.3) is obtained by summing (6.2a) over $i$ and equating the result to the zero matrix - i.e., enforcing the condition $\Sigma_{i} C_{i}=0$. (6.4a) is obtained by multiplying $(6.1 \mathrm{~b})$ by $t_{i} / t$, summing over $i$, and invoking the definitions of $t, \mathbf{b}, \boldsymbol{\mu}$, and $\boldsymbol{\beta}$.

Equations (6.4) define the ubiquitous capital asset pricing model (CAPM), stating that the expected excess return on security $k$ is equal 
to its "beta" times the expected excess return on the market portfolio. However, the parameters appearing in this derivation are all subjective: they are not based on assumptions about an underlying "true" stochastic process or empirically measured returns and covariances, but rather on the aggregated beliefs and risk preferences of the market participants, as revealed in contingent claim prices and wealth distributions. Equation (6.4a) allows us to infer the aggregate vector of expected returns, $\boldsymbol{\mu}$, but only if we independently know the aggregate risk tolerance, $t$, paralleling the result noted above for the individual expected returns.

These convenient analytic results were obtained under strong assumptions about parametric forms of probability distributions and utility functions, but the same qualitative relationships would be expected to hold in practice: agents whose expectations deviated from those of the market would take long or short positions in the primary securities, while agents whose covariances (estimated volatilities) deviated from those of the market would take positions in options; and, other things being equal, agents with higher risk tolerances would choose to bear more risk. However, it would be difficult to separate the absolute magnitude of an agent's risk tolerance from the absolute magnitude of her deviation from market beliefs, and neither the risk-neutral probabilities nor the wealth distribution of an individual agent would be observable with great precision.

\section{ARBITRAGE AND EQUILIBRIUM IN AN EXCHANGE ECONOMY}

The securities market considered in the preceding section is a special kind of exchange economy, characterized by a single commodity (money) and uncertainty about states of the world. A basic theorem of classical welfare economics holds that, in more general multicommodity exchange economies, an allocation of wealth is optimal (jointly utility-maximizing) if and only if there exists a price system with respect to which it is competitive. (In the securities market model, the competitive price system happens to take the form of a risk-neutral distribution.) It was shown by Arrow (1951) and Debreu 
(1954) that this result should be proved using linear programming rather than traditional calculus-based methods in order to take account of non-negativity constraints on consumption. Gale and Mas-Colell (1977) observe that such duality-based proofs do not require the assumption that preferences are complete and transitive (and hence representable by utility functions), merely that they are convex. In this section, we will show that the basic theorem identifying optimality with the existence of a competitive price system can be viewed (not surprisingly) as another application of the arbitrage principle: if preferences are operationally defined in terms of willingness to trade, then Pareto optimality becomes synonymous with the absence of arbitrage opportunities. The relation between no-arbitrage and the existence of a competitive equilibrium has also been discussed by Kreps (1981) and Werner (1987), although those authors define arbitrage opportunities with respect to a given price system, rather than in terms of direct commodity trades. Here, prices emerge as variables in the dual problem, as in the Arrow-Debreu formulation.

Consider an economy with finitely many agents and finitely many types of commodities, where commodity 1 is money. All commodities are considered to have non-negative intrinsic worth (i.e., more is preferred to less) and money is assumed to have positive worth (more money is strictly preferred to less). Suppose we find the economy in a state where agent $i$ 's wealth allocation is $\mathbf{w}_{i}$, a vector whose $j$ th element, $w_{i j}$, is the number of units she holds of commodity $j$. Furthermore, suppose that each agent has asserted preferences for various other wealth positions relative to her current position, meaning that she would be willing to trade her current wealth position for one of the others. In particular, agent $i$ has asserted that $\mathbf{w}_{i}+\mathbf{x}_{i k}$ is preferred to $\mathbf{w}_{i}$, for $k=1,2, \ldots$, where $\mathbf{x}_{i k}$ is a vector of changes in commodity holdings whose $j$ th element is $x_{i k j} . \mathbf{x}_{i k}$ will be called an acceptable trade for agent $i$. (We make no assumption that these trades reflect the "complete and true" preferences of agent $i$, merely that they summarize the extent to which her preferences relative to her current wealth position have been publicly revealed.) Finally, assume that the set of acceptable trades for each agent is convex ${ }^{13}$ - i.e., agent $i$ also prefers $\mathbf{w}_{i}+\Sigma_{k} \alpha_{i k} \mathbf{x}_{i k}$ to $\mathbf{w}_{i}$ for any non-negative $\alpha_{i k}$ such that $\Sigma_{k} \alpha_{i k} \leqslant 1$. Within this framework, we adopt the following: 
DEFINITIONS. A marginal utility vector for agent $i$ is a vector $\mathbf{u}_{i}$ satisfying $\mathbf{u}_{i}^{\mathrm{T}} \mathbf{x} \geqslant 0$ for every trade $\mathbf{x}$ acceptable to her. (That is, $\mathbf{u}_{i}$ is the normal vector of a supporting hyperplane for her set of acceptable trades.) An arbitrage opportunity is a sequence of acceptable trades with some or all agents such that:

(i) each agent ends up with non-negative holdings of each commodity;

(ii) the aggregate amount of each commodity held by the agents is not increased;

(iii) the aggregate amount of money held by the agents is decreased.

A state of the economy which does not admit arbitrage opportunities is Pareto optimal-at least insofar as can be determined from the revealed preferences - and we expect to find the economy in such a state if only because the activities of a small number of arbitrageurs would suffice to drive it there. By linear duality we immediately obtain:

THEOREM 3. There are no arbitrage opportunities if and only if there exist a price vector $\mathbf{p}$ and marginal utility vectors $\left\{\mathbf{u}_{i}\right\}$ for all agents such that:

(i) $p_{1}=1$ (money is the numeraire of prices and marginal utilities);

(ii) $0 \leqslant u_{i j} \leqslant p_{j}$ for every commodity $j$ and every agent $i$ (no agent's marginal utility for a commodity exceeds its price);

(iii) $u_{i j}=p_{j}$ if $w_{i j}>0$ (an agent's marginal utility for a commodity equals its price if she holds a positive quantity of it).

Proof: The arbitrageur's problem is described by the following linear program:

Maximize $z$ over all non-negative $\left\{\alpha_{i k}\right\}$ and unrestricted $z$ subject to $z+\sum_{i k} \alpha_{i k} x_{i k 1}=0$ ( $z$ is the amount of money extracted)

$$
\begin{gathered}
\sum_{i k} \alpha_{i k} x_{i k j} \leqslant 0 \quad \forall j \geqslant 2 \quad \begin{array}{c}
\text { (aggregate commodity holdings do } \\
\text { not increase) }
\end{array} \\
\sum_{k} \alpha_{i k} x_{i k j} \geqslant-w_{i j} \quad \begin{aligned}
\forall i, j \text { (each agent ends up with non- } \\
\text { negative holdings) }
\end{aligned} \\
\sum_{k} \alpha_{i k} \leqslant 1 \quad \forall i \text { (convexity). }
\end{gathered}
$$


Arbitrage is achieved if the optimal objective value is positive. The corresponding dual program is:

$$
\begin{array}{ll}
\text { Minimize } & \sum_{i} v_{i}+\sum_{i j} w_{i j} q_{i j} \text { over all non-negative }\left\{p_{j}\right\},\left\{v_{i}\right\},\left\{q_{i j}\right\} \\
\text { subject to } & v_{i}+\sum_{j}\left(p_{j}-q_{i j}\right) x_{i k j} \geqslant 0 \quad \forall i, k \\
& p_{1}=1 .
\end{array}
$$

The optimal objective value is zero in the dual solution (hence arbitrage is not achievable) if and only if (i) $q_{i j}=0$ wherever $w_{i j}>0$, and (ii) all the $\left\{v_{i}\right\}$ are zero, in which case the dual constraints become:

$$
\sum_{j}\left(p_{j}-q_{i j}\right) x_{i k j} \geqslant 0 \quad \forall i, k
$$

Defining $u_{i j} \equiv p_{j}-q_{i j}$, the conditions of the theorem follow.

Thus, the economy is arbitrage-free if and only if the existing wealth allocation is a competitive equilibrium with respect to some system of prices and some specification of marginal utilities supporting the agents' revealed preferences: no agent can increase her utility by purchasing more of any commodities at those prices and financing the transaction (if necessary) through sales of commodities she already possesses.

Notice that this result neither requires the completeness and transitivity of preferences, nor does it even require any specification of preferences in the vicinity of allocations other than the observed equilibrium allocation. Once again, the essential rationality postulate is a no-arbitrage condition which applies at the market level, and the result obtained is that individual agents must appear to be acting in a way which is consistent with utility-maximization, regardless of their "true" motivations.

In the manner of Debreu (1959), uncertainty can be introduced into the exchange-economy model by defining commodities as physical goods contingent on states of the world. In this setting the marginal utilities are replaced by state-dependent marginal utilities, which may 
be interpreted as products of "true" marginal utilities and state probabilities, a generalization of the concept of risk-neutral probabilities to the multi-commodity setting.

\section{ARBITRAGE AND EQUILIBRIUM IN NONCOOPERATIVE GAMES}

In a recent discussion paper on the interrelationships of finance and economics, Ross (1987) comments on the intrusion of game-theoretic ideas (e.g., bidding, signalling, agency theory, etc.) into the finance arena and expresses discomfort at the "dilution of the arbitrage intuition in many applications of the economic theory of information to problems of modeling financial markets." However, if we examine the foundations of game theory more closely, we once again find the arbitrage principle staring back at us. The notion that strategic rationality is in some way essentially different from individual rationality, or that a strategic equilibrium has deeper structure than a competitive equilibrium, turns out to be largely illusory.

The theory of noncooperative games takes for granted that the rules of the game and the rationality of the players are (somehow) common knowledge. The standard solution concept, Nash equilibrium, is defined as a profile of strategies which are best responses to each other and which are also statistically independent. Aumann (1974) objects to the independence requirement and proposes the alternative concept of correlated equilibrium, which is similar to Nash equilibrium except that it allows randomized strategies to be correlated between players. A distinction can be be made between objective correlated equilibrium, in which the players' beliefs about exogenous events are homogeneous, and subjective correlated equilibrium, in which they are not.

Recently, Aumann (1987) has asserted that objective correlated equilibrium is "the" expression of Bayesian rationality in games because it precisely captures the idea that Bayesian rationality on the part of all players will be common knowledge in every outcome of the game, provided that one accepts the Harsanyi doctrine (Harsanyi 1967) of homogenous prior beliefs. Support for Aumann's claim is provided by Nau and McCardie (1990) who show that the objective correlated equilibrium concept follows from a no-arbitrage argument - essential- 
ly, a game-theoretic version of de Finetti's theorem. Nau (1991a) extends this result to games of incomplete information. The general structure of these results is as follows.

Let common knowledge of the rules of the game be operationalized by supposing that the players' subjective probabilities and payoff functions are publicly revealed - or at least affirmed - via the acceptance of small monetary gambles. Their probabilities for exogenous states of nature are revealed through the acceptance of belief gambles (i.e., the purchase and sale of lottery tickets) exactly as discussed in Section 2. If arbitrage is to be avoided, as has been shown, the outcome of this process must be a "representative" risk-neutral distribution (or set of distributions), which is an apparent common prior. The Harsanyi doctrine is therefore reinterpreted to apply to riskneutral probabilities, not true probabilities.

The players' payoff functions are revealed by their acceptance of preference gambles, which are conditioned on their own choices of strategies and information states, and which yield increments of utility proportional to the differences in utility they perceive between the chosen strategy and other strategies not chosen. The intuitive justification for this is as follows: if player $i$ is observed to play strategy $s_{i}$ given that her information state ("type") is $t_{i}$, then whatever her beliefs about the actions of nature and her opponents, she must feel that the conditional expected utility of playing $s_{i}$ given information $t_{i}$ is greater than or equal to the conditional expected utility of playing any other strategy, say $s_{i}^{\prime}$. In this case, a monetary gamble whose payoff vector (indexed by nature's and her opponent's actions) yields increments of utility proportional to the differences in utility between $s_{i}$ and $s_{i}^{\prime}$ must have non-negative conditional expected utility, and hence it should be acceptable. If the player's marginal utility for money is constant across outcomes of the game, then the monetary payoffs of such a gamble are simply proportional to her true utility differences. If her marginal utility for money is not constant, then the payoffs are proportional to utility differences divided by marginal utilities for money. In the latter case, what is revealed is not the player's "true" payoff function, but rather a "risk-neutral payoff function." (Nau 1991bc)

Now suppose that all players have accepted gambles which are consistent with their beliefs and payoff functions in the ways just 
described, ${ }^{14}$ and consider the position of an outside observer who attempts to construct an arbitrage portfolio. That is, the observer attempts to find a combination of acceptable gambles yielding a gain to himself (i.e., an aggregate loss to the players) which is non-negative in all outcomes of the game and strictly positive in as many outcomes as possible. Such an observer cannot lose, but may win, depending on the play of the game. An outcome in which the observer does not win - i.e., which does not allow arbitrage - is one that we designate as jointly coherent. The corresponding duality theorems (Nau and McCardle 1990, Nau 1991ac) show that the jointly coherent outcomes are precisely those which occur with positive probability in objective correlated equilibria of the revealed game - i.e., the game defined by the risk-neutral probabilities and risk-neutral payoff functions.

The avoidance of arbitrage opportunities requires the players to act as if they had implemented an objective correlated equilibrium of the revealed game. This is not to say that the players must use a correlation device: correlation may exist only in the eyes of the observer if, for example, the players actually intend to play one of several Nash equilibrium strategies but have not bothered to reveal which one. On the other hand, correlation is never ruled out a priori in situations where it would appear to be beneficial. In the case where the revealed game is not the "true" game-i.e., where the players' revealed probabilities and utilities have been distorted by outcome-dependent marginal utilities for money - every objective correlated equilibrium of the revealed game corresponds to a subjective correlated of the true game, in which the players' probabilities may differ (Nau 1991c). Hence, the characterization of a correlated equilibrium as "objective" or "subjective" depends to some extent on the omniscience of the observer. The objective correlated equilibrium concept applies to the revealed game, while the subjective correlated equilibrium concept applies to the true game, but these lead to the same result -i.e., the same set of jointly coherent outcomes.

Far from being a hindrance to measurement, the market again serves as a medium through which requisite knowledge of probabilities and utilities can be disseminated, and the fact that the observable quantities may be risk-neutral rather than "true" values is ultimately unimportant. With the elicitation of the game's rules endogenized in 
this way, strategic equilibrium becomes synonymous with the avoidance of arbitrage in a contingent-claims market whose distinctive feature is that the events are partly under the control of the agents. The arbitrage principle thereby provides a direct link between the concept of competitive market equilibrium and the noncooperative game-theoretic concept of correlated equilibrium, whereas competitive equilibrium has traditionally been viewed either as a close cousin of the Nash equilibrium concept (via the role of fixed-point theorems), or else as a limiting case of the cooperative concept of the core of a game.

The fact that our basic rationality principle leads straight to correlated equilibrium raises questions about the additional assumptions (statistical independence even where correlation would be beneficial, robustness to irrational out-of-equilibrium behavior, etc.) that characterize the stronger Nash concept and its refinements. These assumptions are, for the most part, not operational-they are a priori unenforceable or else place implausible demands on the cognitive powers of the players - and they are precisely the points on which game theory departs from the "arbitrage intuition" underlying the modern theory of financial markets. Of course, an objection which might be raised against the no-arbitrage characterization of strategic rationality is that (as in decision analysis) the hypothesized market usually does not exist. Yet, if such a market does not exist at least in a virtual sense -i.e., if the players are unable even to imagine such a market and visualize their behavior in it - then the traditional gametheoretic assumption that they dwell in a state of numerically precise common knowledge of each other's probabilities and utilities appears dubious. Common knowledge of probabilities, payoffs, and utility functions would imply, among other things, common knowledge of whether-or-not the preplay allocation of state-dependent wealth is an equilibrium. If it is, the assumption of a "virtual market" is innocuous; if it is not, the requirement that the players' selection of strategies should itself constitute any kind of equilibrium appears inconsistent.

\section{CONCLUSION}

It has been shown that the arbitrage principle is more fundamental to Bayesian decision theory, market theory, and game theory than is 
commonly appreciated; that decision analysis and game theory can be informed by ideas of risk-neutral probabilities which originated in the theory of capital markets; that competitive markets serve not only to efficiently allocate production and consumption but also to articulate the subjective parameters of common knowledge; and that economic equilibrium models can survive without the assumption that agents have sharply defined probability distributions and utility functions with respect to which they consciously optimize. No-arbitrage is a realistic standard of rationality which is consistent with a view that optimization is carried out at the market level rather than the agent level, and that decision-making behavior evolves by a process of natural selection. This is not to absolve the individual of the responsibility to use intelligence and foresight, but it suggests that she will be able to do this most successfully within the comparatively narrow field of events with which she is most familiar, for which she has acquired appropriate decision-making rules shaped by the selective process.

\section{ACKNOWLEDGEMENTS}

The authors are grateful for comments on earlier drafts of this paper by Doug Foster, Campbell Harvey, Mike Hemler, Jim Smith, Bob Winkler, workshop participants at FRB Atlanta/Emory University and Virginia Tech, and an anonymous referee. This research was supported by the Business Associates Fund at the Fuqua School of Business.

\section{NOTES}

1 De Finetti's 1974 book begins with the preface: "PROBABILITY DOES NOT EXIST. The abandonment of superstitious beliefs about the existence of Phlogiston, the Cosmic Ether, Absolute Space and Time, ... or Fairies and Witches, was an essential step along the road to scientific thinking. Probability, too, if regarded as something endowed with some kind of objective existence, is no less a misleading conception, an illusory attempt to exteriorize or materialize our true probabilistic beliefs."

${ }^{2}$ The same label is sometimes applied to de Finetti's representation theorem for probability distributions on sequences of exchangeable events, also appearing in the 1937 paper.

${ }^{3}$ The explicit restriction of gambling transactions to small amounts of money distinguishes our treatment from de Finetti's, and admits the possibility that the agent is 
risk-averse or risk-seeking. Small gambles are viewed here as the infinitesimal measurements through which the broad outlines of beliefs and preferences are revealed. A more thorough discussion of this point is given by Nau (1991b).

${ }^{4}$ Here the symbol $\mathbf{E}$ is used interchangeably to denote the name of an event and also the corresponding indicator vector. Thus, $\mathbf{E}-p$ is the payoff vector whose element in the $\theta$ position is $E(\theta)-p$, where $E(\theta)=1$ if the event $\mathbf{E}$ includes the state $\theta$, and $E(\theta)=0$ otherwise.

5 The representative agent is in practice a composite of real agents: the representative agent's buying and selling prices are the maximum advertised buying price and minimum advertised selling price, respectively, among all real agents. This point will be taken up in more detail in Section 4.

${ }^{6}$ Varian (1987) observes: "One of the major advances in financial economics in the past two decades has been to clarify and formalize the exact meaning of 'no arbitrage' and apply this idea systematically to uncover hidden relationships in asset prices. Many important results of financial economics are based squarely on the hypothesis of no arbitrage, and it serves as one of the most basic unifying principles of the study of financial markets." For a rigorous synthesis of arbitrage results, see Garman (1979). ${ }^{7}$ In this tradition, Suppes (1976) asserts: "to insist that we assign sharp probability values to all of our beliefs is a mistake and a kind of Bayesian intellectual imperialism ... [T]here seems to have been a 'natural line of theological succession' from the early belief that God ran the universe in a definite fashion, to the Laplacian belief that the universe ran itself in a definite fashion to [the Bayesian] belief that we all have access to a unique prior probability."

${ }^{8}$ More generally, we could permit consumption in year 1 and then consider utility to be a function of both year- 1 consumption and year- 2 wealth. In the solution of the joint consumption/investment problem, the decision-maker's marginal rate of substitution of wealth between periods would necessarily be brought into equilibrium with the market interest rate.

${ }^{9}$ Howard (1988) presents an eloquent defense of subjective expected utility theory as a guide to action in principle, but his survey of the state-of-the-art in decision analysis is notable for its emphasis on an evolving tool kit of qualitative decision aids: strategygeneration tables, decision quality checklists, influence diagrams, tornado diagrams, normative expert systems. On the quantification of utility, he notes: "While the ability to capture risk preference is an important part of our conceptual view of decision-making, I find it is a matter of real practical concern in only 5 percent to 10 percent of business decision problems. Of course, the situations that require risk preference, such as bidding or portfolio problems, use it seriously. In working with these problems, we have found both the use of generic forms for risk preferences and experience with similar situations to be most helpful." [Emphasis added]

${ }^{10}$ Quadratic dependence of wealth on the primary security returns can be achieved by purchasing "straddles" composed of uniform distributions of call [put] options at all exercise prices greater [less] than the current security price.

${ }^{11}$ The role of contingent claims in the revelation of continuous risk-neutral distributions is described by Breeden and Litzenburger (1978). The basic result is as follows: let $C_{k}(X, T)$ denote the price at time 0 of an option to purchase one share of security $k$ for price $X$ at time $T$. (That is, $X$ is the exercise price of the option and $T$ is its time to maturity.) Then the risk-neutral probability density of the event that the security price is equal to $x$ at time $T$ is proportional to the second derivative of $C_{k}(X, T)$ with respect to 
$X$, evaluated at $X=x$. If options can be written at arbitrary exercise prices on arbitrary portfolios (e.g., mutual funds) of different securities, then in principle the marginal distribution of any function of the state $\theta$ can be uniquely determined. Of course in practice this degree of market completeness is not realized: options are typically traded only at exercise prices which are multiples of $\$ 5$, and only a minority of stocks and mutual funds support liquid options markets.

${ }_{12}$ Notice that (6.3) weights the contribution of agent i's mean vector in proportion to the product of her risk tolerance and her precision matrix, which intuitively measures her willingness to bear risk. If we denote this product as $H_{i}=t_{i} \$_{i}^{-1}$ and $H=t^{\dagger}$ for the individual and the market, respectively, then the aggregation formulas take the simple form:

$$
H=\sum_{i} H_{i}, \quad \text { and } \quad H \mu=\sum_{i} H_{i} \mu_{i} .
$$

The same aggregation formulas for means and covariances are obtained in the absence of contingent claims markets - i.e., when agents' wealth distributions are required to be linear in the primary security returns (Lintner 1969) - although the market risk-neutral distribution is not directly observable in this case. More general aggregation formulas based on the exponential utility assumption, incorporating arbitrary probability distributions and heterogenous time preferences, are given by Huang and Litzenberger (1988, pp. 146-147).

${ }^{13}$ The linear and additivity assumptions are replaced here by the convexity assumption, which is more familiar in this context: it explicitly admits the possibility of "large" trades among risk-averse traders, although only small trades are needed for the arbitrage argument.

14 Although the acceptance of such gambles can be viewed as a canonical medium of preplay communication among the players, we hasten to point out that once the rules of the game have become common knowledge - by whatever means - the gambles have no bearing on the play of the game: their acceptability must itself be common knowledge at this point, and hence of no strategic importance.

\section{REFERENCES}

Allais, M.: 1953 'Le Comportement de l'Homme Rationnel devant le Risque: Critique des Postulats et Axiomes de l'Ecole Américaine', Econometrica 53, 503-546.

Allais, M.: 1971 'Les Théories de l'Equilibre Economique Général et de l'Efficacité Maximale: Impasses Récents et Nouvelles Perspectives', Revue d'Economie Politique, May-June 1971. Translated and reprinted in G. Schwödiauer (ed.) (1978) Equilibrium and Disequilibrium in Economic Theory, D. Reidel, Dordrecht, Holland.

Arrow, K. J.:1951 'An Extension of the Basic Theorems of Classical Welfare Economics', Proceedings of the Second Berkeley Symposium on Mathematical Statistics and Probability, University of California Press.

Aumann, R.: 1962 'Utility Theory Without the Completeness Axiom', Econometrica 30, 445-462.

Aumann, R.: 1974 'Subjectivity and Correlation in Randomized Games', J. Math. Econ. 1, 67-96. 
Aumann, R.: 1987 'Correlated Equilibrium as an Expression of Bayesian Rationality', Econometrica 55, 1-18.

Banz, R. and M. Miller: 1978 'Prices for State-Contingent Claims: Some Estimates and Applications', J. Business 51(4), 653-672.

Beja, Avraham: 1967 'Capital Markets with Delayed "Learning"', Ph.D. Thesis, Stanford University.

Black, F. and M. Scholes: 1973 'The Pricing of Options and Corporate Liabilities', J. Political Economy 81, 637-654.

Breeden, D. and R. Litzenberger: 1978 'Prices of State Contingent Claims Implicit in Options Prices', J. Business 51, 621-652.

Brennan, M.: 1979 'The Pricing of Contingent Claims in Discrete Time Markets', $J$. Finance 24(1), 53-68.

Callen, H. B.: 1960 Thermodynamics, Wiley, New York.

Copeland, T., T. Koller, and J. Murrin: 1990 Valuation: Measuring and Managing the Value of Companies, Wiley, New York.

Cornfield, J.: 1969 'The Bayesian Outlook and Its Application', Ann. Stat. 4, 1051-1064.

Cox, J. and S. Ross: 1976 'The Valuation of Options for Alternative Stochastic Process', J. Financial Econ. 3(1/2), 145-166.

Debreu, G.: 1954 'Valuation Equilibrium and Pareto Optimum', Proc. Nat. Acad. Sci. 588-592.

Debreu, G.: 1959 Theory of Value, Wiley, New York.

de Finetti, B.: 1937 'La Prévision: Ses Lois Logiques, Ses Sources Subjectives', Ann. Inst. Henri Poincaré 7, 1-68. Translation reprinted in H. E. Kyburg and H. E. Smokler (eds.) (1980) Studies in Subjective Probability, 2nd ed., Robert Krieger, New York, 53-118.

de Finetti, B.: 1974 Theory of Probability, Vol. 1, Wiley, New York.

Drèze, J.: 1970 'Market Allocation Under Uncertainty', European Econ. Rev. 2, $133-165$.

Ellsberg, D.: 1961 'Risk, Ambiguity, and the Savage Axioms', Quarterly. J. Econ. 75, 643-669.

Freedman, D. and R. Purves: 1969 'Bayes' Method for Bookies', Ann. Math. Stat. 40, 1419-1429.

Gale, D.: 1960 The Theory of Linear Economic Models, McGraw-Hill, New York.

Gale, D. and A. Mas-Colell: 1977 'On the Role of Complete, Transitive Preferences in Equilibrium Theory', in G. Schwödiauer (ed.) (1978) Equilibrium and Disequilibrium in Economic Theory, D. Reidel, Dordrecht, Holland, 7-14.

Garman, M.: 1979 'A Synthesis of the Pure Theory of Arbitrage', Working Paper No. 98. Institute of Business and Economic Research, University of California, Berkeley.

Good, I. J.: 1962 'Subjective Probability as the Measure of a Non-Measurable Set', in E. Nagel, P. Suppes, and A. Tarski (eds.) Logic, Methodology, and Philosophy of Science, Stanford University Press. Reprinted in H. E. Kyburg and H. E. Smokler (eds.) (1980) Studies in Subjective Probability, 2nd ed., Robert Krieger, New York, 133-146.

Harsanyi, J.: 1967 'Games with Incomplete Information Played by 'Bayesian' Players: I-III', Management Sci. 14, 159-182, 320-334, 486-502.

Heath, D. and W. Sudderth (1972) 'On a theorem of De Finetti, Odds making, and Game Theory', Ann. Math. Stat. 43, 2072-2077. 
Hogarth, R. and M. Reder, eds.: 1986 Rational Choice, University of Chicago Press. Also in $J$. Business 59(4), 2 (Supplement to October 1986 issue).

Howard, R. A.: 1988 'Decision Analysis: Practice and Promise', Manag. Sci. 34(6), 679-695.

Huang, C. F. and R. H. Litzenberger: 1988 Foundations for Financial Economics, North-Holland, New York.

Kadane, J. B. and R. L. Winkler: 1988 'Separating Probability Elicitation from Utilities', J. Amer. Stat. Assoc. 83, 357-363.

Keynes, J. M.: 1921 Treatise on Probability, in The Collected Writings, Vol. VIII, edited by D. Moggridge, MacMillan, London, 1973.

Koopman, B. O.: 1940 'Axioms and Algebra of Intuitive Probability', Ann. Math. 41, 269-292.

Kreps, D.: 1981 'Arbitrage and Equilibrium in Economies with Infinitely Many Commodities', J. Math. Econ. 8, 15-35.

Levi, I.: 1980 The Enterprise of Knowledge, MIT Press, Cambridge, Massachusetts.

Lindley, D.: 1972 Bayesian Statistics: A Review, Society for Industrial and Applied Mathematics, Philadelphia.

Lintner, J.: 1969 'The Aggregation of Investors' Diverse Judgments and Preferences in Purely Competitive Security Markets', J. Financial \& Quantitative Analysis 4(4), 346-400.

Merton, R.: 1973 'The Theory of Rational Option Pricing', Bell Journal of Economics and Management Science 4, 141-183.

Modigliani, F. and M. Miller: 1958 'The Cost of Capital, Corporation Finance, and the Theory of Investment', Amer. Econ. Rev. 48, 261-297.

Munier, B.: 1991 'Market Uncertainty and Belief Formation with a Finite Number of Events', $J$. Risk and Uncertainty, forthcoming.

Nash, J.: 1951 'Non-cooperative Games', Ann. Math. 54, 286-295.

Nau, R. and K. McCardle: 1990 'Coherent Behavior in Noncooperative Games', J. of Econ. Theory 50(2), 424-444.

Nau, R.: 1990 'The Incoherence of Agreeing to Disagree', Fuqua School of Business Working Paper 9006.

Nau, R.: 1991a 'Joint Coherence in Games of Incomplete Information', Management Science, forthcoming.

Nau, R.: 1991b 'Coherent Decision Analysis with Inseparable Probabilities and Utilities', Fuqua School of Business Working Paper 9101.

Nau, R.: 1991c 'The Relativity of Subjective and Objective Correlated Equilibria', Fuqua School of Business Working Paper 9102.

Nelson, R. and S. Winter: 1982 An Evolutionary Theory of Economic Change, Belknap Press, Cambridge.

Pierce, D.: 1973 'On Some Difficulties in a Frequency Theory of Inference', Ann. Stat. 1, 241-250.

Ross, S.: 1976 'The Arbitrage Theory of Capital Asset Pricing', J. Econ. Theory 13, 341-360.

Ross, S.: 1978 'A Simple Approach to the Valuation of Risky Streams', J. Business 51(3), 453-475.

Ross, S.: 1987 'The Interrelations of Finance and Economics: Theoretical Perspectives', Amer. Econ. Rev. 77(2), 29-34. 
Rubinstein, M.: 1976 'The Valuation of Uncertain Income Streams and the Pricing of Options', Bell J. Economics and Management Science 7, 407-425.

Savage, L.,: 1954 The Foundations of Statistics, Wiley, New York.

Scarf, H.: 1990 'Mathematical Programming and Economic Theory', Operns. Research 38(3), 377-385.

Seidenfeld, T.: 1988 'Decision Theory Without "Independence" or Without "Ordering:" What's the Difference?, Economics and Philosophy 4, 267-315.

Simon, H. A.: 1955 'A Behavioral Model of Rational Choice', Quart. J. Econ. 59, 99-118.

Simon, H. A.: 1976 'From Substantive to Procedural Rationality', in S. J. Latsis (ed.), Method and Appraisal in Economics, Cambridge University Press, Cambridge. Reprinted in H. A. Simon, Models of Bounded Rationality, MIT Press, Cambridge MA, 1982.

Smith, C. A. B.: 1961 'Consistency in Statistical Inference and Decision', J. Roy. Stat. Soc. B 23; 1-25.

Suppes, P.: 1974 'The Measurement of Belief', J. Roy. Stat. Soc. B 36, 160-175.

Suppes, P.: 1976 'Testing Theories and Foundations of Statistics' (with discussion), in W. L. Harper and C. A. Hooker (eds.), Foundations of Probability Theory, Statistical Inference, and Statistical Theories of Science, Vol. II, D. Reidel, Dordrecht, Holland, 437-455.

Trigeorgis, L. and S. P. Mason: 1987 'Valuing Managerial Flexibility', Midland Corporate Finance Journal 5, 14-21.

Varian, H.: 1987 'The Arbitrage Principle in Financial Economics', J. Economic Perspectives 1, 55-72.

Walley, P.: 1991 Statistical Reasoning with Imprecise Probabilities, Chapman and Hall, London.

Werner, J.: 1987 'Arbitrage and the Existence of Competitive Equilibria', Econometrica 55, 1403-1418.

Wilson, R.: 1968 'The Theory of Syndicates', Econometrica 36(1), 119-132.

Yaari, M.: 1985 On the Role of "Dutch Books" in the Theory of Choice Under Risk. Nancy Schwarz Memorial Lecture, Northwestern University.

The Fuqua School of Business,

Duke University,

Durham, NC 27706,

U.S.A. 